\title{
Hipopituitarismo postraumático: reporte de un caso
}

\section{Carlos Alfonso Builes Barrera', Juliana Sierra Zuluaga ${ }^{2}$}

${ }^{1}$ Endocrinólogo de Adultos. Hospital Universitario San Vicente Fundación. Docente de la Universidad de Antioquia-sección de Endocrinología. Grupo de Endocrinología y Metabolismo.

${ }^{2}$ Especialista en Medicina Interna. Médica Residente de

Endocrinología y Metabolismo, Universidad de Antioquia.

Correspondencia: endocrinobuiles@gmail.com

Hospital Universitario San Vicente Fundación

Bloque 6. Oficina de Endocrinología y Diabetes

Fecha de recepción: 18/09/2015

Fecha de aceptación: 30/09/2015

\section{Resumen}

Se presenta el caso de un paciente con diagnóstico de novo de hipopituitarismo, cuya manifestación inicial fue con hiponatremia grave, secundaria a una insuficiencia suprarrenal central. Adicionalmente se documentó hipotiroidismo e hipogonadismo centrales. El paciente tuvo una lesión cerebral traumática cuatro décadas atrás y se encontraron en la resonancia nuclear de hipófisis hallazgos de hipoplasia hipofisaria y aracnoidocele selar, apoyando el diagnóstico de hipopituitarismo anterior. El caso resalta la presentación de hipopituitarismo diagnosticado décadas después de una lesión cerebral traumática. Se propone un enfoque para el diagnóstico, tratamiento y seguimiento de estos pacientes en nuestro medio.

Palabras claves: lesión cerebral traumática, hipopituitarismo, hiponatremia, disfunción neuroendocrina.

\section{Abstract}

We present the case of a patient with previously undiagnosed hypopituitarism, it presents whose initial manifestation was a severe hyponatremia secondary to a central adrenal insufficiency, and besides this deficit he had central hypothyroidism and hypogonadism. In his past medical history he had a moderate traumatic brain injury almost 40 years before. The case highlights the presentation of hypopituitarism decades after a traumatic brain injury. An approach to the diagnosis, treatment and follow-up of these patients in our environment is proposed.

Keywords: traumatic brain injury, hypopituitarism, hyponatremia, neuroendocrine dysfunction.

\section{Introducción}

La lesión cerebral traumática (LCT) es un problema de salud pública reconocido en todo el mundo, y es una causa de mortalidad y discapacidad a largo plazo. Las principales causas de LCT son los accidentes de tránsito $(50 \%$ de todos los casos), caídas, incidentes relacionados con violencia, trauma de cabeza relacionados con el deporte (hockey, fútbol, boxeo, clavados y kickboxing) y los accidentes relacionados con la guerra, incluyendo lesiones por explosión ${ }^{(1)}$.

La LCT se había considerado previamente como una causa rara de hipopituitarismo; sin embargo, en los últimos 15 años se ha reportado un aumento en la prevalencia de la disfunción neuroendocrina, siendo tan frecuente como del $31 \%$ a un año ${ }^{(2-9)}$. La frecuencia de hipopituitarismo crónico (definido como una duración de al menos tres meses) después de LCT varía ampliamente entre los estudios, con rangos entre 15\% y 50\%. Esta variación en la disfunción de la hipófisis podría explicarse por diferentes factores como la gravedad, tipo y tiempo transcurrido del trauma, la población estudiada, el diseño del estudio, las pruebas endocrinas y los criterios utilizados para el diagnóstico de las deficiencias de hormonas de la hipófisis.

La Escala de Coma de Glasgow es la herramienta clínica más utilizada para evaluar la gravedad de la lesión cerebral ${ }^{(10)}$. La probabilidad de desarrollar hipopituitarismo se incrementa entre más severa es la LCT, pero puede presentarse aún en formas leves.

La deficiencia hormonal postraumática puede ser aislada o múltiple, y parcial o completa. Las hormonas más frecuentemente comprometidas en su orden son hormona del crecimiento (GH), gonadotropinas (FSH/LH), hormona adrenocorticotropa (ACTH) y hormona estimulante de tiroides (TSH) ${ }^{(4,8)}$.

\section{Presentación del caso}

Hombre de 69 años de edad, con cuadro clínico de un mes de evolución de debilidad generalizada, vértigo, vómito y dolor abdominal difuso. En los últimos días, los síntomas se habían empeorado por lo que consultó al servicio de urgencias.

Como antecedentes personales conocidos tenía hipertensión arterial y dislipidemia, sin consumo de medicación regular, ni de diuréticos tiazídicos al momento de la consulta. Sin antecedentes familiares relevantes de hipotiroidismo primario, diabetes o enfermedad endocrina.

$\mathrm{Al}$ examen físico se encontró presión arterial (PA) de $132 / 61 \mathrm{mmHg}$, pulso de $78 \mathrm{lpm}$, pérdida de la cola de las cejas, edema bipalpebral, escaso vello facial y axilar, tiroides de tamaño y consistencia normal. Ginecomastia bilateral sin se- 
creción al exprimir. Abdomen blando sin dolor a la palpación. Testículos blandos, de tamaño normal y vello púbico con distribución normal. Piel sin hiperpigmentación. Edema blando, hasta tercio distal de las extremidades inferiores. Inestabilidad para la marcha, reflejos osteotendinosos disminuidos en forma bilateral.

Ante el predominio de los síntomas vertiginosos e inestabilidad para la marcha, se enfoca el cuadro clínico como un síndrome de fosa posterior y se solicitan paraclínicos, incluyendo una angiorresonancia cerebral contrastada. Se documenta hiponatremia grave, con TSH dentro de rangos normales y atrofia cerebral de predominio frontotemporal y hallazgos sugestivos de leucoencefalopatía microangiopática moderada, sin alteraciones en la circulación en la angiorresonancia.

En el estudio de la hiponatremia se documentó el valor bajo de cortisol sérico, en un paciente sin antecedente conocido reciente de uso de esteroides tópicos, orales, inhalados o musculares. Se obtuvo un valor bajo de ACTH y de T4 libre, lo que orientó al diagnóstico de hipopituitarismo anterior (tabla 1).

Tabla 1. Exámenes de laboratorio iniciales

\begin{tabular}{l|c}
\hline Potasio $\mathrm{mEq} / \mathrm{L}$ & 3,65 \\
\hline Sodio $\mathrm{mEq} / \mathrm{L}$ & 118 \\
\hline Calcio $\mathrm{mg} / \mathrm{dl}$ & 8,8 \\
\hline Albúmina $\mathrm{gr} / \mathrm{dl}$ & 3,97 \\
\hline Fósforo $\mathrm{mg} / \mathrm{dl}$ & 2,5 \\
\hline Creatinina $\mathrm{mg} / \mathrm{dL}$ & 1,44 \\
\hline BUN $\mathrm{mg} / \mathrm{dL}$ & 10 \\
\hline TSH $\mathrm{mUI} / \mathrm{L}$ & 2,52 \\
\hline
\end{tabular}

Se solicitó valoración por endocrinología por las deficiencias hormonales documentadas. Al reinterrogar, el paciente refirió somnolencia excesiva, disminución de la libido, disminución en la frecuencia de la necesidad de afeitarse, intolerancia al frio e intolerancia al ejercicio. En términos generales el paciente había presentado una disminución global progresiva en su funcionalidad.

Se confirmó el diagnóstico de hipopituitarismo anterior con el perfil hormonal completo (tabla 2) y la resonancia de silla turca mostró marcado adelgazamiento de la hipófisis y aracnoidocele selar (figura 1).

Cuarenta años atrás, el paciente había sufrido una lesión traumática cerebral moderada con un elemento contundente en un hecho violento. Presentó pérdida del conocimiento por unos minutos y cefalea posterior, sin requerir asistencia médica en ese momento. En los años posteriores al trauma no refiere haber percibido algún cambio en su vida. Se casó y tuvo hijos.

Se inició tratamiento intrahospitalario con hidrocortisona 50 miligramos intravenosos cada seis horas y posteriormen-
Tabla 2. Perfil hormonal completo y perfil lipídico

\begin{tabular}{l|c|c}
\multicolumn{1}{c|}{ Laboratorio } & Valor & Rango \\
\hline Cortisol $\mu \mathrm{g} / \mathrm{dL}$ & 0,7 & $5-20$ \\
\hline Prolactina $\mathrm{ng} / \mathrm{mL}$ & 13,7 & $3,4-19$ \\
\hline ACTH $\mathrm{pg} / \mathrm{ml}$ & $<5$ & $0-46$ \\
\hline Testosterona total $\mathrm{ng} / \mathrm{mL}$ & 1,19 & $1,56-5,63$ \\
\hline FSH IU/L & 3,59 & $1-12$ \\
\hline LH IU/L & 5,81 & $2-12$ \\
\hline Somatomedina C ng/mL & 103,7 & $64-188$ \\
\hline TSH mUI/L & 2,52 & $0,35-4,9$ \\
\hline T4L ng/dL & 0,48 & $0,7-1,48$ \\
\hline LDL mg/dL & 148 & \\
\hline Triglicéridos mg/dL & 324 & \\
\hline
\end{tabular}

$\mu \mathrm{g}$ : microgramos, dL: decilitro, ng: nanogramos, mg: miligramos, pg: picogramos, FSH: hormona folículo estimulante, LH: hormona luteinizante, TSH: hormona estimulante de la tiroides, T4: tetrayodotiroxina, L: litro, UI: unidades internacionales LDL: colesterol de baja densidad.

Figura 1. RMN de silla turca. Corte sagital secuencia SE T1. Silla turca de diámetro AP normal. Aracnoidocele selar (flecha horizontal). Parénquima hipofisiario que no supera los $2 \mathrm{~mm}$ de diámetro (flecha vertical)

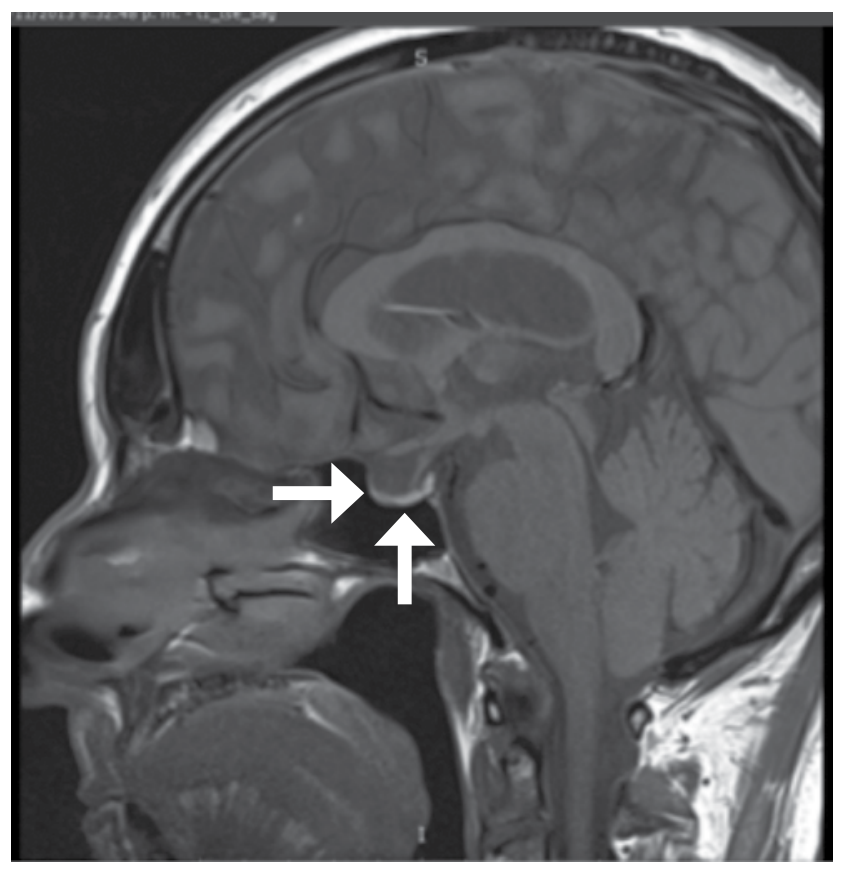

te se adicionó levotiroxina 25 microgramos vía oral cada día con franca mejoría de sus síntomas neurológicos. La hiponatremia se corrigió completamente y en forma ambulatoria se 
inició suplencia con enantato de testosterona con muy buena evolución clínica y mejoría notoria en su calidad de vida. Actualmente recibe prednisolona 5 miligramos vía oral al día, levotiroxina 50 microgramos vía oral al día, enantato de testosterona 250 miligramos intramuscular cada tres semanas y atorvastatina $20 \mathrm{mg}$ vía oral cada noche, con muy buena adherencia y tolerancia a los medicamentos.

\section{Discusión}

El hipopituitarismo postraumático o hipopituitarismo inducido luego de una LCT fue descrito por primera vez en $1918^{(11)}$, pero sólo ha cobrado importancia en la comunidad médica en los últimos 15 años, dados los resultados de estudios retrospectivos y prospectivos que muestran una mayor prevalencia entre el 15\%-50\%, y una mayor asociación con discapacidad física y psicológica, con cambios cognitivos, comportamentales y sociales a largo plazo ${ }^{(2-9)}$. La falta de asociación clínica con el antecedente traumático craneano, la poca familiarización del médico familiar con el diagnóstico de hipopituitarismo y los increíbles mecanismos de adaptación corporal al déficit hormonal crónico puede explicar el tiempo de latencia tan prolongado para el diagnóstico del paciente.

La frecuencia de la disfunción de la hipófisis varía de acuerdo con múltiples factores, la mayoría de los estudios establecen la gravedad de la LTC según la Escala de Coma de Glasgow $^{(10)}$ y permiten clasificarla como leve (puntaje de 13-15), moderada (puntaje de 9-12) o grave (puntaje de 3-8), siendo la variable más determinante. En una revisión sistemática de 14 estudios $^{(2)}$ que agrupó 1.014 pacientes, la prevalencia del hipopituitarismo fue del 35\%, 10,9\% y 16,8\% para pacientes con LCT severa, moderada y leve, respectivamente. Entre 809 pacientes que fueron evaluados cinco meses después de la lesión, las deficiencias de la hormona del crecimiento y de gonadotropinas fueron las más prevalentes, afectando cerca del $12 \%$ de los pacientes cada una, seguida por el déficit de ACTH y de TSH en $8 \%$ y 4\%, respectivamente. En otra revisión sistemática de 66 estudios con 5.386 pacientes adultos la prevalencia de hipopituitarismo a un año fue del 31,6\%, siendo los grupos de más alto riesgo aquellos que sufrieron fracturas de la base del cráneo y LCT severa. Sin embargo, esta revisión tiene alto grado de heterogeneidad en los estudios ${ }^{(9)}$. Aunque la LCT reportada por el paciente fue leve, en el paciente se documentó déficit de los ejes tirotropo, corticotropo y gonadotropo, para los cuales recibió suplencia.

Los datos sobre la historia natural del hipopituitarismo inducido por LCT son limitados. La mayoría de los estudios incluyen periodos de seguimiento variables entre tres meses y cinco años. Se estima que la frecuencia de hipopituitarismo se reduce con el tiempo y que muchos de los pacientes recuperan su función hormonal. Sin embargo, un gran número de pacientes presentan persistencia de la disfunción de la hipófisis, empeoramiento del déficit, o incluso desarrollan nuevas deficiencias(6-8). Esto ha sido reportado con periodos de latencia tan largos como 30 años después de la LCT $^{(12)}$.

La fisiopatología de la disfunción pituitaria después de la LTC aún no se entiende completamente. Factores como el traumatismo mecánico directo, el insulto vascular, el daño hipotalámico, los cambios inflamatorios, la compresión por la hemorragia, el edema o aumento de la presión intracraneal, la predisposición genética y la autoinmunidad pueden jugar un papel en el desarrollo de anormalidades neuroendocrinas. Por su localización anatómica, el hipotálamo, el tallo y la hipófisis son estructuras vulnerables a estos fenómenos. Los vasos portales largos pasan a través del diafragma de la silla turca y pueden dañarse después de un trauma en la cabeza con más facilidad ${ }^{(13)}$. Estudios recientes revelaron que la autoinmunidad puede tener algún papel en el desarrollo y/o empeoramiento del hipopituitarismo. Se han reportado anticuerpos antihipófisis y anticuerpos antihipotalámicos en títulos altos en pacientes con hipopituitarismo luego de $\mathrm{LCT}^{(8-13)}$.

Todo paciente con LCT moderada o grave debe ser evaluado durante la fase aguda y en el curso crónico de su recuperación. Hay pacientes con LCT leve que cumplen criterios de riesgo para desarrollar hipopituitarismo y deben ser estudiados de igual forma. Se incluyen pacientes con necesidad de hospitalización durante más de 24 horas, necesidad de vigilancia en unidad de cuidados intensivos, necesidad de procedimientos neuroquirúrgicos, amnesia mayor a 30 minutos, y cambios anatómicos en las neuroimagenes iniciales. Independientemente de la gravedad de la lesión, si un paciente presenta signos y síntomas de hipopituitarismo en cualquier momento luego del evento, debe ser evaluado. En los pacientes que luego del trauma quedan en estado crónico vegetativo con poca expectativa de vida no se requieren estudios complementarios hormonales ${ }^{(14)}$.

En las dos primeras semanas posteriores a la LCT, la atención debe centrarse en el eje corticosuprarrenal (comprometido hasta en el $50 \%$ de pacientes) y la función de la hipófisis posterior. En los meses siguientes se hará énfasis en todos los ejes de la hipófisis anterior y posterior ${ }^{(15)}$.

Los valores de cortisol sérico menores de $3 \mu \mathrm{g} / \mathrm{dL}$ en ayunas apoyan fuertemente el diagnóstico de insuficiencia suprarrenal, mientras que los valores de cortisol sérico mayores a $18 \mu \mathrm{g} / \mathrm{dL}$ descartan disfunción del eje hipotálamo-hipófisissuprarrenal. Pacientes con valores intermedios (3,1-17,9 $\mu \mathrm{g}$ / dL) también pueden tener hipocortisolismo y deben ser considerados como candidatos para terapia con sustitución con glucocorticoides en presencia de hallazgos clínicos sugestivos. Estos pacientes requieren pruebas dinámicas para evaluar completamente la función del eje en la fase crónica. En la fase aguda es poco práctico realizar estas pruebas dinámicas dada la variación fisiológica que sufre este eje por enfermedad gra- 
ve, además de no contar con puntos de corte definidos para el cortisol sérico. Se ha propuesto que todos los pacientes hospitalizados con LCT moderada o severa y cortisol sérico menor de $10 \mu \mathrm{g} / \mathrm{dL}$ podrían suplementarse con glucocorticoides durante la fase aguda ${ }^{(14,15)}$ y posteriormente realizar prueba de estimulación con ACTH para definir necesidad de suplencia continua en forma crónica.

La diabetes insípida central (DIC) debe sospecharse ante la presencia de poliuria (producción de orina $>200 \mathrm{~mL} /$ hora durante al menos dos horas o de $40 \mathrm{ml} / \mathrm{kg} / 24$ horas (> 3 litros/ 24 horas en pacientes de peso promedio) y/o hipernatremia. Estos pacientes están en riesgo de deshidratación si su sensorio está alterado, y carecen de acceso al agua. Entre los pacientes con hipernatremia que no reciben diuréticos o manitol la presencia de una orina inapropiadamente diluida ( $<700 \mathrm{mOsm} / \mathrm{kg}$ ) es coherente con el diagnóstico de DI. La prueba de deshidratación puede ayudar a establecer el diagnóstico de la DIC y diferenciarla de la DI nefrogénica o polidipsia primaria de acuerdo con la respuesta en la osmolaridad urinaria luego de aplicación de desmopresina. Esta prueba sólo debe realizarse en pacientes estables en un entorno supervisado y que no tengan hipernatremia. La medición de otros parámetros como arginina vasopresina o copeptina en plasma tiene utilidad diagnóstica pero con dificultades técnicas en entornos clínicos habituales y su uso no está recomendado. En la resonancia magnética de hipófisis, la ausencia de la "mancha brillante posterior" en las secuencias de T1 sin contraste apoya el diagnóstico de DIC ${ }^{(15)}$.

La evaluación hormonal durante la fase crónica se deberá realizar entre 3 a 6 meses de la LCT, y en cualquier momento ante la aparición de clínica sugestiva de hipofunción ${ }^{(14-15)}$. Los pacientes con hipocortisolismo de origen hipofisiario se presentan con fatiga, pérdida de peso, anorexia, mareo, dolor articular varias semanas a meses después de la LCT. A diferencia de la enfermedad de Addison, no cursan con hiperpigmentación ni con hipercaliemia, pues carecen de ACTH y la secreción de aldosterona está preservada. La hiponatremia se atribuye a una secreción inapropiada de hormona antidiurética y al déficit de cortisol. La prueba de estímulo con ACTH para aclarar la respuesta de producción suprarrenal puede verse alterada hasta las 6 u 8 semanas después de la LCT, cuando se va presentando la atrofia cortical por falta de estímulo apropiado de la ACTH. El paciente de este caso presentaba los síntomas descritos y cursaba con hiponatremia grave, hipocortisolismo, ACTH baja, potasio normal, sin hiperpigmentación, apoyando el origen central.

El hipotiroidismo central no se manifiesta durante la fase más aguda de la enfermedad (debido a que la vida media de la tiroxina es cercana a siete días). El perfil tiroideo muestra una T4 libre baja y una TSH inapropiadamente normal o discretamente elevada para el valor bajo de T4 libre como se identificó en el paciente, esto refuerza la necesidad de medir la T4 libre para aclarar la función tiroidea en pacientes con alta sospecha de enfermedad hipofisiaria en los que el valor aislado de la TSH puede causar falsos negativos y omisión del diagnóstico correcto. En los pacientes con enfermedad aguda y grave, el diagnóstico diferencial más importante es con el síndrome de eutiroideo enfermo, también conocido como el síndrome del T3 bajo. En nuestro medio no se dispone de la medición de T3 reversa que apoyaría este diagnóstico en caso de hallarse elevada. En general, se recomienda hacer la evaluación del eje tiroideo sólo 4 a 6 semanas después del evento agudo para evitar confusiones.

La evaluación del eje hipofisiario gonadal debe realizarse en pacientes estables durante la fase de rehabilitación después de la LCT, pues en la fase aguda generalmente está suprimida. El desarrollo de amenorrea secundaria en la mujer o de disminución de la libido y de caracteres sexuales secundarios masculinos junto con un valor repetido de testosterona total bajo, y valores inapropiadamente normales de FSH y LH apoyan el diagnóstico de hipogonadismo central como se hizo con el paciente. Adicionalmente, el perfil de hipogonadismo hipogonadotrófico puede producirse por hiperprolactinemia, que se reporta en cerca del $11 \%$ de los $\operatorname{casos}^{(3)}$.

La evaluación de la secreción de hormona del crecimiento (HC) debe realizarse varios meses después de la LCT y sólo en aquellos pacientes cuyas otras deficiencias hormonales han sido corregidas. En pacientes adultos, la deficiencia de HC puede producir poca energía y capacidad para el ejercicio, alteración en la calidad de vida, adiposidad central, dislipidemia, resistencia a la insulina, baja masa ósea, alteración de la memoria y concentración. No se recomienda realizar mediciones aleatorias de HC para evaluar la secreción hormonal, pues muchas personas sanas tienen niveles de $\mathrm{HC}$ indetectables a lo largo del día. Los niveles del factor de crecimiento similar a la insulina tipo 1 (IGF-1) carecen de sensibilidad en el diagnóstico. Sin embargo, en pacientes con antecedente de LCT y niveles bajos de IGF-1 para la edad y sexo y presencia de deficiencias hormonales hipofisiarias ( $\geq 3$ ) es muy probable que tengan déficit de HC y no necesitarían una prueba formal con inducción de hipoglucemia o estímulo con arginina o glucagón. En todos los otros casos se necesitaría de una prueba de estímulo para hacer el diagnóstico de deficiencia de HC.

Los pacientes con hipopituitarismo deben tener una resonancia magnética nuclear para descartar la presencia de una lesión selar ${ }^{(15)}$. En el caso, el hallazgo de aracnoidocele y tamaño hipofisiario menor de $2 \mathrm{~mm}$, confirma la falta de tejido hipofisiario, acorde con el diagnóstico de hipopituitarismo y posible falta de irrigación sanguínea de la hipófisis anterior posterior a la LCT.

En pacientes críticamente enfermos con hipocortisolismo se recomienda dar dosis de estrés de hidrocortisona entre 50 a 100 miligramos intravenosos cada ocho horas. En pacientes estables debe suplementarse con hidrocortisona oral 15 a 25 
miligramos vía oral al día fraccionado en 2 a 3 dosis o prednisolona 2,5 a 5 miligramos al día; no es necesario dar suplencia mineralocorticoide porque la secreción de aldosterona está preservada.

Los pacientes con hipotiroidismo central pueden necesitar cerca de 1,3 a 1,6 microgramos por kilogramo al día de levotiroxina, aunque en pacientes ancianos o con larga evolución de los síntomas podría usarse inicialmente una dosis menor. En caso de insuficiencia adrenal asociada, debe suplementarse inicialmente el esteroide y luego la levotiroxina para evitar desencadenar una crisis de insuficiencia suprarrenal. Esta recomendación se realizó con el paciente con buena respuesta. El seguimiento debe hacerse con T4 libre, buscando que se encuentre en la mitad del rango de referencia y titulando según la evolución clínica.

Los pacientes con hipogonadismo pueden recibir remplazo con esteroides sexuales si no está contraindicado. Se debe tener especial consideración al remplazar la testosterona en los hombres con enfermedad cardiovascular por los posibles efectos adversos en este sistema.

Algunos estudios han mostrado un posible efecto benéfico al dar remplazo en dosis bajas de hormona del crecimiento en la cognición, calidad de vida, composición corporal con aumento de la masa ósea y muscular y disminución de la grasa visceral, mejoría en el perfil de lípidos y capacidad del ejer- cicio. Se recomienda usar dosis bajas 0,2 miligramos al día y 0,3 miligramos al día, subcutáneos, para hombres y mujeres, respectivamente, para lograr niveles de IGF-1 entre 0 a +1 desviación estándar para el sexo y edad ${ }^{(15)}$.

La LCT es una causa frecuente de hipopituitarismo crónico. Los pacientes se presentan con las mismas manifestaciones que aquellos con hipopituitarismo atribuible a otras causas; sin embargo, dado que la clínica puede ser muy sutil y fácilmente atribuible a la misma LCT, es probable que la mayoría de los pacientes con hipopituitarismo permanezcan sin diagnosticar y, por lo tanto, no se traten. Se recomienda medir luego de 3 a 6 meses y al año de la LCT (especialmente en aquellos con TEC moderado a severo por escala de Glasgow): TSH, T4 libre, sodio sérico, cortisol sérico, somatomedina C (IGF-1) y niveles de FSH/estradiol para mujeres o LH/testosterona total en hombres en la búsqueda intencionada de hipopituitarismo.

La sospecha clínica y especialmente el interrogatorio dirigido al antecedente de LCT y la búsqueda intencional de hipopituitarismo en pacientes con LCT moderada o severa tienen un papel crítico en el diagnóstico oportuno para de esta manera poder ofrecer el tratamiento adecuado con el fin de mejorar los resultados en estos pacientes.

Conflictos de interés: Ninguno que declarar para la escritura del texto.

\section{Referencias}

1. Coronado VG, Xu L, Basavaraju SV, et al. Surveillance for traumatic brain injury-related deaths--United States, 1997-2007. MMWR SurveillSumm. 2011; 60:1-32

2. Schneider HJ, Kreitschmann-Andermahr I, Ghigo E, Stalla GK, Agha A. Hypothalamus pituitary dysfunction following traumatic brain injury and aneurysmal subarachnoid hemorrhage: a systematic review. JAMA. 2007; 298: 1429-1438.

3. Agha A, Rogers B, Sherlock M, et al. Anterior pituitary dysfunction in survivors of traumatic brain injury. J ClinEndocrinolMetab. 2004; 89(10):4929-4936.

4. Aimaretti G, Ambrosio MR, Di Somma C, et al. Residual pituitary function after brain injury-induced hypopituitarism: a prospective 12-month study. J ClinEndocrinolMetab. 2005; 90(11):6085-6092.

5. Schneider HJ, Schneider M, Saller B, et al. Prevalence of anterior pituitary insufficiency 3 and 12 months after traumatic brain injury. Eur J Endocrinol. 2006; 154(2):259-265.

6. Tanriverdi F, Senyurek H, Unluhizarci K, Selcuklu A, Casanueva FF, Kelestimur F. High risk of hypopituitarism after traumatic brain injury: a prospective investigation of anterior pituitary function in the acute phase and 12 months after trauma. J ClinEndocrinolMetab. 2006;91(6): 2105-2111.

7. Tanriverdi F, Ulutabanca H, Unluhizarci K, Selcuklu A, Casanueva FF, Kelestimur F. Three years prospective investigation of anterior pituitary function after traumatic brain injury: a pilot study. ClinEndocrinol (Oxf). 2008; 68(4):573-579.
8. Tanriverdi F, De Bellis A, Ulutabanca H, et al. A five year prospective investigation of anterior pituitary function after traumatic brain injury: is hypopituitarism long-term after head trauma associated with autoimmunity? J Neurotrauma. 2013; 30(16):1426-1433.

9. Lauzier F, Turgeon AF, Boutin A, et al. Clinical outcomes, predictors, and prevalence of anterior pituitary disorders following traumatic brain injury: a systematic review. CritCareMed. 2014;42:712-721.

10. Teasdale G, Jennett B. Assessment of coma and impaired consciousness. A practical scale. Lancet. 1974; 2(7872):81-84.

11. Cryan E. Pituitary damage due to skull base fracture. DtschMedWochenschr. 1918; 44:1261.

12. Streetz-van der Werf C, Karges W, Blaum M, ..Kreitschmann-Andermahr. Addisonian Crisis after Missed Diagnosis of Posttraumatic Hypopituitarism. J Clin Med. 2015 May; 4(5): 965-969.

13. Tanriverdi F, Schneider HJ, Aimaretti G, Masel BE, Casanueva FF, Kelestimur F. Pituitary dysfunction after traumatic brain injury: a clinical and pathophysiological approach. Endocr Rev. 2015 Jun;36(3):305-42.

14. Tanriverdi F,Kelestimur F. Pituitary dysfunction following traumatic brain injury: clinical perspectives Neuropsychiatr Dis Treat. 2015; 11: 18351843.

15. Tritos N, Yuen K, Kelly D. A neuroendocrine approach to patients with traumatic brain injury. EndocrPract. 2015;21: 823-831. 